Francisco Prieto, Zhangfei Su, J. Jay Leitch, Manuela Rueda, Jacek Lipkowski,

"Quantitative subtractively normalized interfacial Fourier transform infrared reflection spectroscopy study of the adsorption of adenine on Au(111) electrode.", Langmuir, 2016, 37,3827-3835.

\title{
QUANTITATIVE SUBTRACTIVELY NORMALIZED INTERFACIAL FOURIER TRANSFORM INFRARED REFLECTION SPECTROSCOPY STUDY OF THE ADSORPTION OF ADENINE ON AU (111) ELECTRODES
}

\author{
Francisco Prieto $\left.{ }^{a}\right)^{*}$, Zhangfei Su$^{b)}$, J. Jay Leitch ${ }^{b)}$, Manuela Rueda ${ }^{a)}$, Jacek Lipkowskib) \\ a)Department of Physical Chemistry University of Seville. \\ c/ Professor Garcia Gonzalez 2. 41012 Seville. SPAIN \\ b) Electrochemistry Technology Centre. Department of Chemistry. \\ College of Physical \& Engineering Science. University of Guelph \\ Guelph, Ontario, CANADA. N1G 2W1 \\ *dapena@us.es
}

\section{ABSTRACT}

Quantitative substractively normalized interfacial Fourier transform infrared reflection spectroscopy (SNIFTIRS) was used to determine the molecular orientation and identify the metal-molecular interactions responsible for the adsorption of adenine from the bulk electrolyte solution onto the surface of the Au (111) electrode. The recorded $p$ polarized IR spectra of the adsorbed species were subtracted from the collected spolarized IR spectra to remove the IR contributions of the vibrational bands of the desorbed molecules that are located within the thin layer cavity of the spectroelectrochemical cell. The intense IR band around $1640 \mathrm{~cm}^{-1}$, which is assigned to the pyrimidine ring stretching vibrations of the $\mathrm{C} 5-\mathrm{C} 6$ and $\mathrm{C} 6-\mathrm{N} 10$ bonds, and the IR band at $1380 \mathrm{~cm}^{-1}$, which results from a combination of the ring stretching vibration of 
the $\mathrm{C} 5-\mathrm{C} 7$ bond and the in-plane $\mathrm{CH}$ bending vibration, were selected for the quantitative analysis measurements. The transition dipoles of these bands were evaluated by DFT calculations. Their orientations differed by $85 \mp 5^{\circ}$

The tilt angles of adsorbed adenine molecules were calculated from the intensity of these two vibrations at different potentials. The results indicate that the molecular plane is tilted at an angle of $40^{\circ}$ with respect to the surface normal of the electrode and rotates by $16^{\circ}$ around its normal axis with increasing electrode potential. This orientation results from the chemical interaction between the N10 and gold atoms coupled with the $\pi-\pi$ parallel stacking interactions between the adjacent adsorbed molecules. Furthermore, the changes in the molecular plane rotation with the electric field suggest that the $\mathrm{N} 1$ atom of adenine must also participate in the interaction between the molecule and metal.

\section{INTRODUCTION}

The adsorption of DNA bases at solid electrodes is an interesting area of research because the electrode/electrolyte interface can be used to model the electrostatic properties of wide variety of biological interfaces. These studies offer a unique opportunity to investigate the effect of the electric field on the organization of DNA bases and the interactions between their complementary DNA bases. Moreover, these systems have technological interest in the development of new biocompatible materials, biosensors and molecular electronic devices, in addition to gaining a better understanding of the fundamental principles of molecular recognition. ${ }^{1,2,3,4,5,6}$ 
The adsorption of adenine on gold single crystal electrodes of low Miller index planes (Scheme 1) was previously characterized by cyclic voltammetry and capacitance measurements, ${ }^{7}$ which demonstrated that the adenine molecules are weakly chemisorbed to the surface gold atoms and that the adsorption process is strongly affected by the $\mathrm{pH}$ of the bulk and the crystallographic orientation of the metal surface. ${ }^{8}$ In addition, there is also a close relationship between the chemisorption of adenine and the lifting of the reconstruction of the electrode surface. The thermodynamic study of the adenine adsorption on the $A u(111)$ surface from neutral solutions was performed using charge density data, which was obtained by integrating of the chronoamperometric curves. ${ }^{7}$ The current transients were measured by employing a specially designed potential-step program to avoid the influence of the surface reconstruction processes on the total charge. ${ }^{9,} 10$ The value for the free Gibbs energy of adsorption $\left(-35 \mathrm{~kJ} \mathrm{~mol}^{-1}\right)$, obtained via thermodynamic analysis of the charge density data, further supported a weak chemisorption process. The maximum surface excess $\left(3.5 \times 10^{-10} \mathrm{~mol} \mathrm{~cm}{ }^{-2}\right)$ suggested that the molecular plane of adenine adopts a tilted orientation with respect to the surface. ${ }^{7}$

Potential controlled Infrared Reflection Absorption Spectroscopy (IRRAS) 8 , 11, 12 and scanning tunneling microscopy (STM), ${ }^{13}$ were performed in order to obtain microscopic information about the adsorption of adenine on the surface of gold electrodes, such as the nature of intermolecular interactions, orientation of the adenine molecules, in addition to gaining a better understanding about how the molecule is coordinated to the gold atoms on the surface. The electrochemical STM measurements confirmed that adenine induces the lifting of the surface 
reconstruction. The images show that the gold atoms released by lifting the reconstruction of the $\mathrm{Au}(111)$ surface form islands of a monoatomic height that remain "frozen" along the characteristic $\mathrm{Au}(111)$ reconstructed solitons (reconstruction lines). The images also showed that the adenine ad-layer is organized into twisted rows, which are aligned along the three main directions of the $A u(111)$ surface, $[\overline{1}, 1,0],[1,0, \overline{1}]$ and $[0, \overline{1}, 1]$. Each row is formed by molecules stacked every 3-4 angstroms, which is compatible with $\pi$-electronic intermolecular interactions.

The IRRAS studies of the electrosorption of adenine have provided information about the chemical identity of the adsorbed species and nature of the adenine coordination to the metal surface. ${ }^{8}, 11,12$ The IR absorption spectra of adsorbed adenine on gold electrodes display bands that correspond to the in-plane vibrations of the pyrimidine ring. The appearance of this band suggests that adenine adopts a normal or tilted orientation of the molecular plane relative to the electrode surface according to the surface selection rules. The assignments of the observed surface active IR bands in comparison with the spectra published for adenine-metal complexes in the literature, suggests that adenine interacts with the gold electrode via the amine nitrogen atom (N10), in combination with either the pyrimidinic nitrogen (N1) or the imidazolic nitrogen (N7) as presented in Scheme 1. Moreover, the analysis of the spectroelectrochemical data as a function of $\mathrm{pH}^{8,11}$ lead to the conclusion that only the neutral adenine molecule and the N9-deprotonated anionic adenine form can chemically interact with the metal, even at very low $\mathrm{pH}$ where adenine is in its cationic (i.e. protonated at the $\mathrm{N} 1$ atom) form. These results imply that the chemical 
adsorption of adenine induces the deprotonation of the $\mathrm{N} 1$ atom, even at $\mathrm{pH}$ values that are significantly lower than the corresponding pKa value of $4.8 .{ }^{14,15}$

Unfortunately, the analysis of the IRRAS data was qualitative in nature; therefore, the conclusions were merely speculative. Consequently, there are many questions that remain unclear about the structure of the adsorbed adenine layer on the surface of the gold electrodes, which include: How do the N1 or N7 atoms of adenine participate in the interaction with the electrode? What is the actual tilt angle of the molecular plane relative to the electrode surface? How does the tilt angle change with the electrode potential? What is the driving force for this change? Moreover, in the presence of thymine (the complementary base of adenine), ${ }^{16,17}$ the infrared absorption bands suggest that there is a change in the overall orientation of the adsorbed adenine, which may result in a variation in the coordination between adenine and the gold atoms on the electrode surface. In order to determine changes in the molecular orientation, a quantitative evaluation of the tilt angles of the adsorbed adenine is required. In the last decade, several studies have applied SNIFTIRS to gain quantitative information about electrochemically adsorbed layers on single crystal electrodes, which include pyridine on $\mathrm{Au}(110),{ }^{18}$ citrate on $\mathrm{Au}(111),{ }^{19}$ 2mercaptobenzimidazole on $\mathrm{Au}(111),{ }^{20,}{ }^{21}$ or sulfate on $\mathrm{Pt}(111) \cdot{ }^{22}$ All these studies are based on the comparison between the experimental SNIFTIR spectrum of the adsorbed molecular film and a theoretically simulated spectrum of randomly oriented molecules with the same surface concentration. ${ }^{23}$ The integrated intensity of an IR absorbance band of the adsorbed molecular film obtained with p-polarized light, $A_{p \text { (surface) }}$, where the electric field of the photon is parallel to the plane of incidence, is proportional to 
the scalar product of the transition dipole vector and the electric field of the photon according to the following expression: $:^{24,25}$

$$
\int A_{p(\operatorname{surface})} d \bar{v} \propto \Gamma\left|\vec{\mu} \cdot \vec{E}_{p}\right|^{2}=\Gamma|\mu|^{2}\left\langle E_{p}{ }^{2}\right\rangle \cos ^{2} \theta
$$

where $\Gamma$ is the surface concentration of the IR absorbing species, $\theta$ is the angle between the transition dipole moment and the electric field vector of photon, $|\mu|$ is the transition dipole modulus and $\left\langle E_{p}{ }^{2}\right\rangle$ is the mean square electric field strength of the p-polarized radiation at the reflection surface. Equation 1 can also be used to calculate the orientation of an adsorbed film of the same surface concentration with randomly oriented molecules, however, the angle of the transition dipole moment in this case would be equal to the magic angle $\left(\theta=55^{\circ}\right)$. From the ratio of the integrated intensities of the experimental and calculated spectra for randomly adsorbed molecules, it is possible to determine the angle of the transition dipole moment, $\theta$, for the adsorbed adenine on the gold surface.

$$
\frac{\int A_{\text {exp }} d \bar{v}}{\int A_{\text {calc }} d \bar{v}}=\frac{\cos ^{2} \theta}{\cos ^{2} 55^{\circ}}
$$

Knowing the direction of the transition dipole with respect to the molecular coordinates, it is then possible to attain the average orientation of adsorbed molecules on the metal surface. ${ }^{24,25,26}$

The aim of this work is to perform the quantitative SNIFTIRS study of adenine adsorption on the $A u(111)$ electrode from a neutral solution to determine the orientation of adsorbed adenine molecules on the electrode surface. The molecular level information will be used to determine which fragments of the adenine molecule 
$\left(\mathrm{NH}_{2}\right.$ group and/or either $\mathrm{N} 1$ or $\mathrm{N} 7$ heteroatom) are coordinated to the electrode surface and the average tilt angle of the adenine molecular plane with respect to the surface normal. Furthermore, we will show how the electrode charge influences the orientation of molecules at the electrode surface. This work demonstrates the power of quantitative SNIFTIRS studies of organic films at an electrode surface by illustrating that the spectroscopic data not only provides unique information regarding structure of the film, but also gives insight into how the molecules are coordinated to the metal surface.

\section{EXPERIMENTAL}

\section{Reagents and electrodes.}

The sodium fluoride (ACS certified, Fisher Scientific) was used as the supporting electrolyte. It was cleaned for 30 minutes in an ozone chamber (UVO cleaner, Jelight, Irvine, CA) prior to use. The adenine (Sigma-Aldrich) was used without further purification. The solutions were prepared from deuterium oxide, $\mathrm{D}_{2} \mathrm{O}(99.9 \%$, Cambridge Isotope Laboratories, Cambridge, MA). The working electrode was a gold $\mathrm{Au}(111)$ oriented single crystal, prepared following the Clavilier method ${ }^{27}$. It was flame annealed before its insertion into the spectroelectrochemical cell. A platinum foil was used as counter electrode and a silver/silver chloride reference electrode. A salt bridge, containing the electrolyte solution, was used to avoid interference from chloride ions present in the reference electrode. The analyte solution in the infrared reflection absorption measurements consisted of $2 \mathrm{mM}$ adenine in $0.5 \mathrm{M} \mathrm{NaF} / \mathrm{D}_{2} \mathrm{O}$. In the transmission measurements, an adenine concentration of $10 \mathrm{mM}$ was dissolved in 
the $0.5 \mathrm{M} \mathrm{NaF} / \mathrm{D}_{2} \mathrm{O}$ electrolyte. Prior to all experiments, the IR cell was cleaned in an acid bath (1:3 mixture of $\mathrm{HNO}_{3}$ and $\mathrm{H}_{2} \mathrm{SO}_{4}$ ) and thoroughly rinsed with Milli-Q UV plus (Millipore, Bedford, MA) water ( $\geq 18.2 \mathrm{M} \Omega \mathrm{cm}$ ). The IR cell was soaked in Milli-Q water for several hours, rinsed again and then dried over night. A HEKA potentiostat (Lambrecht/Pfalz, Germany) was used to apply the desired potential to the cell. The spectra were recorded with a resolution of $4 \mathrm{~cm}^{-1}$ (i.e. spectral data points are computed every $2 \mathrm{~cm}^{-1}$ ). The bands are plotted by interpolation between these points; therefore, the band center position can be determined with uncertainty $0.5 \mathrm{~cm}^{-1} .{ }^{28}$

\section{FT-IR measurements.}

All of the FT-IR spectra have been obtained with a Nicolet 8700 spectrometer equipped with a liquid $\mathrm{N}_{2}$ cooled MCT-A detector. The instrument and tabletop optics module (TOM) were purged with dry $\mathrm{CO}_{2}$-free air from a Puregas heatless dryer (Whatman, Piscataway, NJ). Infrared transmission spectra of the aqueous $\left(\mathrm{H}_{2} \mathrm{O}\right.$ and $\mathrm{D}_{2} \mathrm{O}$ ) adenine solutions $(10 \mathrm{mM})$ in $0.5 \mathrm{M} \mathrm{NaF}$ were measured with a transmission cell that consisted of two plane calcium fluoride windows spaced by a c.a. $50-\mu \mathrm{m}$ PTFE gasket. The exact thickness of the transmission cell was determined from the separation between constructive interference fringes of the transmission spectrum registered in air. ${ }^{29}$

For the infrared reflection absorption experiments, the IR beam was polarized in either the plane of incidence (p-radiation) or the direction perpendicular to the plane of incidence (s-radiation). According to the surface selection rules for metals, ${ }^{30}$ the intensity of the s-polarized radiation is cancelled at the reflection plane, which 
means that only species that are desorbed in the bulk solution of the thin layer cavity between the metal and IR prism are capable of absorbing the infrared radiation. In contrast, the IR absorption of the p-polarized radiation occurs at both the metal electrode surface and in the bulk solution. Furthermore, the electric field of the ppolarized radiation is enhanced upon reflection at the metal surface, which means that p-polarized radiation is more sensitive to surface species than those desorbed in the bulk of solution.

The design of the spectroelectrochemical IR cell has been previously described in the work by Li et al. ${ }^{18}$ The optical set up, according to the design of Faguy, ${ }^{31,32,33,34}$ allows for adjustments of the incidence angle and focal point of the IR beam. The IR transparent window was a ZnSe hemispherical prism with a diameter of $25 \mathrm{~mm}$. The $\mathrm{Au}(111)$ surface was pressed against the IR window to create a thin layer of electrolyte with a thickness of a few micrometers. In order to get the maximum surface enhancement of the p-polarized radiation at the reflection point, the incidence angle of the IR beam has been set just below the critical angle with a focal distance between 9 and $10 \mathrm{~mm}$ from the base of the ZnSe hemisphere prism. The incidence angle of the IR beam and thickness of the electrolyte layer between the optical window and the metal surface have been precisely determined with the Hansen model ${ }^{35}$ where the experimental reflectivity spectrum is compared with a theoretical spectrum that is calculated for three parallel, homogeneous phases (ZnSe | electrolyte | Au) according to the method developed by Li et al. ${ }^{18}$

The SNIFTIR spectra were obtained by collecting the sample reflectivity spectrum $\left(R_{a d s}\right)$ with adsorbed adenine by registering 50 interferograms at a sample 
potential $\left(E_{a d s}\right)$ at which adenine molecules are adsorbed to the gold surface, and the reference reflectivity spectrum $\left(R_{\text {des }}\right)$ by registering the same number of interferograms at a low potential $\left(E_{\text {des }}\right)$ where adenine is fully desorbed from the electrode surface. This collection cycle has been repeated 80 times and the results were averaged in order to increase the signal to noise ratio. The corresponding IR reflection absorption spectrum at a single sample potential is obtained representing $\Delta R / R_{\text {des }}$ vs. wavenumber:

$$
\left(\frac{\Delta R}{R_{\text {des }}}\right)_{S N I}=\frac{R_{\text {des }}-R_{\text {ads }}}{R_{\text {des }}} \approx 2.3 \Gamma\left[\varepsilon\left(E_{\text {des }}\right)-\varepsilon\left(E_{\text {ads }}\right)\right]=2.3 \Delta A
$$

Where $\boldsymbol{\Gamma}$ is the surface concentration of the adsorbed species, $\boldsymbol{\varepsilon}\left(\boldsymbol{E}_{\text {des }}\right)$ and $\boldsymbol{\varepsilon}\left(\boldsymbol{E}_{\text {ads }}\right)$ are the molar absorptivity coefficients of adenine in the completely desorbed and adsorbed states, respectively. As a result, the negative bands in the SNIFTIR spectrum correspond to the IR absorption by molecules that are adsorbed at the electrode surface, while positive bands account for an increase in IR absorption of the molecules located within solution. This increase in IR absorbance is caused by a decrease in the bulk solution concentration due to an adsorption process. The loss in IR absorbance occurs if there is a shift in the frequency of the IR band or if the adsorbed molecule adopts an orientation where the direction of the transition dipole moment for the given vibration is parallel to the metal surface.

The sample potential, $E_{a d s}$, was changed within the limits of adsorption of adenine. The SNIFTIRS measurements, including the potential steps and collection of the interferograms, were driven by the macro utility program within the OMNIC ${ }^{T M}$ Spectral Software package (Thermo Scientific, Waltham, MA, USA) on a computer 
equipped with a digital to analog converter (PIO DA4 board, ICP DAS CO,Hsinchu, Taiwan).

\section{DFT calculations of the vibrational transitions.}

The directions of the transition dipole of each vibration have been obtained by DFT geometry optimization using a Unix version of Gaussian 09 on a Fujitsu Primergy cluster. The vibrational calculations were performed with the B3LYP/ 6-31++G(d,p) functional and basis set as previously described by Giese and McNaughton in their study of adenine adsorption on silver surfaces ${ }^{36}$.

\section{RESULTS AND DISCUSSION}

\section{FT-IR spectra of adenine in solutions of neutral pH.}

Figure 1 displays the IR transmission spectra of the $1800-1300 \mathrm{~cm}^{-1}$ region for $10 \mathrm{mM}$ adenine/0.5 M NaF electrolyte solutions prepared in $\mathrm{D}_{2} \mathrm{O}$ (Figure 1a) and $\mathrm{H}_{2} \mathrm{O}$ (Figure 1b). All of the spectral bands within this frequency range correspond to the in-plane vibrations, which are associated with the $\mathrm{NH}_{2}$ scissoring and skeletal stretching vibrations. $^{36}$

\section{- Figure 1 -}

The hydrogen atoms that are associated with the nitrogen groups of adenine readily exchange with deuterium from the $0.5 \mathrm{M} \mathrm{NaF} / \mathrm{D}_{2} \mathrm{O}$ electrolyte. The IR absorbance for the deuterated adenine shows the appearance of an intense band around $1630 \mathrm{~cm}^{-1}$, which is associated with the in-plane pyrimidine ring stretching 
vibrations of the $\mathrm{C} 5-\mathrm{C} 6$ and $\mathrm{C} 6-\mathrm{N} 10$ bonds. In the $\mathrm{H}_{2} \mathrm{O}$ electrolyte, the pyrimidinic ring stretching band $\left(1639 \mathrm{~cm}^{-1}\right)$ overlaps with the scissoring vibrational band $\left(1652 \mathrm{~cm}^{-1}\right)$ of the amine group. In deuterium oxide, the bending vibrational mode of the amine group is shifted to lower frequency $\left(\sim 1200 \mathrm{~cm}^{-1}\right)$, while the pyrimidinic ring stretching vibration remains within the $1600 \mathrm{~cm}^{-1}$ region, which completely resolves these two independent vibration bands. Additionally, the strong $\mathrm{OH}$ bending mode of water is also active in the $1600 \mathrm{~cm}^{-1}$ region whereas, the OD bending mode is shifted to 1200 $\mathrm{cm}^{-1}$. Therefore, the quantitative SNIFTIRS analysis has only been performed on the spectral data obtained in deuterium oxide to provide a more accurate measurement of the IR intensity of the pyrimidine ring vibration by ensuring that this IR band is free from all spectral interferences.

\section{Quantitative SNIFTIRS of adenine adsorbed on Au(111) electrode.}

The p-polarized SNIFTIR spectra of the $\mathrm{Au}(111)$ electrode in a $0.5 \mathrm{M} \mathrm{NaF} / \mathrm{D}_{2} \mathrm{O}$ electrolyte solution containing $2 \mathrm{mM}$ of adenine at given sample potentials $\left(E_{\text {ads }}\right)$ are presented in Figure 2. The negative-going component of the IR band at $1640 \mathrm{~cm}^{-1}$ is enhanced and shifted to slightly higher wavenumbers compared to the positive-going portion of the band. The SNIFTIR spectrum obtained using s-polarized photons at a sample potential of $0.09 \mathrm{~V}$ is also included in Figure 2 . This spectrum contains only the positive band at $1639 \mathrm{~cm}^{-1}$ due to a decrease in the concentration of the desorbed adenine molecules within the thin layer cavity. A comparison of this s-polarized band and the transmission IR spectrum with the positive part of the bipolar band in the SNIFTIRS spectrum recorded using p-polarized radiation at the same sample potential 
confirms that the positive lobe of the bipolar band does in fact correspond to the absorbance by desorbed molecules.

- $\quad$ Figure 2 -

The high signal-to-noise ratio of the intense negative band makes it ideal for accurately calculating the angle between its transition dipole and the normal direction to the electrode. The direction of the transition dipole of this vibration is nearly parallel to $\mathrm{C} 4-\mathrm{C} 5$ bond direction. However, two different transitions dipole angles are needed in order to determine the orientation of the molecule plane relative to the electrode surface. For this purpose, the band appearing in the SNIFTIR spectra at 1380 $\mathrm{cm}^{-1}$ was also selected for further quantitative analysis. According to DFT calculations, this IR band is assigned to the ring stretching vibrations (mainly C5-N7) and the inplane $\mathrm{CH}$ bending mode. This vibration involves a transition dipole moment that is oriented in a different direction (approximately the same direction of the C5-N7 bond) and provides sufficient intensity to allow for quantitative analysis. According to the DFT calculations, the angle between the directions of transition dipoles of the bands at $1640 \mathrm{~cm}^{-1}$ and at $1380 \mathrm{~cm}^{-1}$ is equal to $85 \pm 5$ degrees.

To obtain the orientation of adsorbed molecules, the IR contribution of the species desorbed in the solution must be removed from the experimental p-polarized SNFTIR spectrum using the following procedure. First, the s-polarized SNIFTIR spectra are recorded at each sample potential. However, the average mean square electric field strengths of the s-polarized, $\left\langle E_{s}{ }^{2}\right\rangle$, and p-polarized $\left\langle E_{p}{ }^{2}\right\rangle$, radiation within the thin layer cavity are not equal. Therefore, the magnitude of the electric field strength of the 
experimental s-polarized SNIFTIR spectrum, $\left(\frac{\Delta R}{R_{d e s}}\right)_{s_{(\exp )}}$, must be normalized with that of the p-polarized spectrum using the following correction:

$$
\left(\frac{\Delta R}{R_{\text {des }}}\right)_{S_{(n o r m)}}=\left(\frac{\Delta R}{R_{\text {des }}}\right)_{S_{(\exp )}} \times \frac{\left\langle E_{p}{ }^{2}\right\rangle}{\left\langle E_{S}{ }^{2}\right\rangle}
$$

The average mean square electric field strengths within the thin layer cavity of the spectroelectrochemical cell can be evaluated on the basis of Fresnel equations provided that the angle incidence and thickness of the electrolyte are known. ${ }^{18,} 34,37$ These two parameters can be determined using the procedure described in reference 18. The normalized s-polarized SNIFTIR spectra can then be used to remove the IR contribution of the desorbed adenine molecules from the p-polarized SNIFTIR spectra to give the IR absorption spectra of the adsorbed adenine molecules:

$$
\left(\frac{\Delta R}{R_{\text {des }}}\right)_{p_{(\mathrm{ads})}}=\left(\frac{\Delta R}{R_{\text {des }}}\right)_{p_{(\mathrm{exp})}}-\left(\frac{\Delta R}{R_{\text {des }}}\right)_{S_{(\mathrm{norm})}}
$$

An example of this correction procedure is presented in Figure 3a) for both the 1380 and $1640 \mathrm{~cm}^{-1}$ spectral bands at a potential of $-0.11 \mathrm{~V} \mathrm{vs.} \mathrm{Ag} /$.

-Figure 3

As previously mentioned, the quantitative SNIFTIRS analysis is performed by calculating the ratio of the integrated areas of a vibrational band in the experimental spectrum of adsorbed molecules and the theoretical spectrum of a film of randomly oriented molecules of the same surface concentration according to equation (2):

$$
\frac{\int A_{p_{\mathrm{ads}}} d \bar{v}}{\int A_{p_{\text {calc }}} d \bar{v}}=\frac{\int\left(\frac{\Delta R}{R_{\text {des }}}\right)_{p_{\mathrm{ads}}} d \bar{v}}{\int\left(\frac{\Delta R}{R_{\text {des }}}\right)_{p_{\text {calc }}} d \bar{v}}=\frac{\cos ^{2} \theta}{\cos ^{2} 55^{\circ}}
$$


An example of the theoretical p-polarized spectrum is shown in Figure $3 b)$. To calculate the area of the theoretical IR absorption band of a film of randomly oriented molecules, the surface excesses of the adsorbate as a function of the potential must be obtained from thermodynamic measurements. This data has been previously obtained in the thermodynamic study of the adsorption/desorption process of adenine on $\mathrm{Au}(111)$ electrodes from neutral solutions. ${ }^{7}$

Additionally, isotropic optical constants, which consist of the refractive index $\left(n_{D 3 A d}\right)$ and attenuation coefficient $\left(k_{D 3 A d}\right)$, of deuterated adenine molecules are required to calculate the simulated spectrum at the frequencies of the selected IR bands. These parameters can be obtained from the IR transmission spectrum of deuterated adenine by an iterative procedure described in a previous work. ${ }^{18}$ The optical constants of the 1380 and $1640 \mathrm{~cm}^{-1}$ vibrations of deuterated adenine, obtained from the IR transmission spectrum presented in Figure 1a), are shown in Figure 4.

- $\quad$ Figure 4-

The adenine optical constants are used to generate the IR absorption spectrum of an adenine film with randomly oriented molecules at the maximum surface concentration, $\Gamma_{\max }$, at $0.2 \mathrm{~V}$. At all other surface concentrations $(\Gamma)$, the simulated spectra can be obtained applying a factor of $\Gamma / \Gamma_{\max }$.

The integrated absorbance intensities of the 1380 and $1640 \mathrm{~cm}^{-1}$ vibrational bands from both the simulated and experimental spectrum were used to calculate the angles between the normal direction to the electrode and the transition dipoles of the 1640 
$\mathrm{cm}^{-1}\left(\theta_{1640}\right)$ and $1380 \mathrm{~cm}^{-1}\left(\theta_{1380}\right)$ vibrations according to Equation (6). The calculated transition dipole angles are plotted as a function of the electrode potential in Figure 5.

- Figure 5 -

Figure 5 shows that $\theta_{1640}$ increases from $40^{\circ}$ to a limiting value of $52^{\circ}$ at a potential of $0.2 \mathrm{~V}$. This suggests that the transition dipole of the $1640 \mathrm{~cm}^{-1}$ vibration adopts an orientation that becomes more parallel with the gold electrode surface. In contrast, $\theta_{1380}$ decreases from an initial angle of $72^{\circ}$ to a limiting value of $63^{\circ}$ as the electrode potential becomes more positive. Therefore, the transition dipole of the $1380 \mathrm{~cm}^{-1}$ vibration becomes more normal to the electrode surface at more positive potentials.

The angle $\theta_{\bar{v}}$ between the transition dipole moment of an in-plane vibration and the normal direction to the electrode includes contributions from two individual angles: i) the angle between the in-plane transition dipole with respect to the projected component of the surface normal of the molecular plane for the in plane vibration, $\varphi_{\bar{v}}$ and ii) the angle between the normal of the electrode surface and the molecular plane, which is referred to as the molecular tilt angle $\alpha$, and is common for all in plane vibrations. The decomposition of the individual angles associated with an adsorbed adenine molecule is presented in Scheme 1.

- Scheme 1 -

From the geometric reasoning provided in the supporting information, it can be deduced that:

$$
\begin{aligned}
\cos \theta_{1640} & =\cos \alpha \times \cos \varphi_{1640} \\
\cos \theta_{1380} & =\cos \alpha \times \cos \varphi_{1380}
\end{aligned}
$$


With the aid of these equations and by taking the sum $\varphi_{1640}+\varphi_{1380}=85 \pm 5^{\circ}$, the values of the tilt angle of the molecular plane, $\alpha$, and the rotation angles of the transition dipoles of the absorption bands at $1640 \mathrm{~cm}^{-1}$ and $1380 \mathrm{~cm}^{-1}, \varphi_{1640}$ and $\varphi_{1380}$, can be calculated. These values are plotted against the electrode potential in Figure 6.

- Figure 6 -

The tilt angle $\alpha$ of the adsorbed adenine molecular plane in Figure 6a is nearly independent of the applied electrode potential with an average value of $\sim 40^{\circ}$. This inclination of the molecular plane is in good agreement with the conclusions of the thermodynamic study that suggested a tilted orientation of the molecular plane. ${ }^{7}$ It is also consistent with the structure observed in the electrochemical STM images of adenine adlayers on $\mathrm{Au}(111)$ electrodes, ${ }^{13}$ where the reported distance between the adenine molecules was found to be between 3 to 4 angstroms. This distance is too short to correspond to intermolecular hydrogen bonding or van der Waals interactions, but does fall within the expected distances for parallel $\pi$-stacking interactions. The inclination of molecular planes found from the SNIFTIRS analysis indicates that this $\pi-\pi$ interactions must be of the "parallel displaced" type, 38,39 instead of the "parallel-sandwich" type. Assuming that the distance between adjacent adsorbed molecules at the surface is of $3.5 \AA$ with a slope of $40^{\circ}$, the effective displacement is only $2.3 \AA$. In the case of solid adenine, crystallographic structure measurements ${ }^{14}$ concluded that the molecules are stacked parallel-displaced with the non-overlapping dipoles as a consequence of a twisting film structure. The tilt angle of the adenine adsorbed molecules can be facilitated by the $\mathrm{sp}^{3}$ hybridization of the 
amine nitrogen $\mathrm{N} 10 .^{7}$ This hybridization was also predicted by other authors, ${ }^{40,41}$ who studied the coordination of adenine to metals on the basis of ab-initio calculations and suggest that the $\mathrm{sp}^{3}$ hybridization of the $\mathrm{N} 10$ nitrogen facilitates the adsorption to the metal.

In Figure $6 \mathrm{~b}$ plots the values of $\varphi_{1640}$ and $\varphi_{1380}$ as a function of the electrode potential. The data suggests that as the potential increases from -0.3 to $0.2 \mathrm{~V}$ vs. $\mathrm{Ag} / \mathrm{AgCl}, \varphi_{1640}$ increases from $18^{\circ}$ to a limiting value of $34^{\circ}$ and $\varphi_{1380}$ decreases from $66^{\circ}$ to $50^{\circ}$. These changes are caused by a $16^{\circ}$ rotation of the molecular plane around its normal axis. This rotation is modeled in Scheme 2 and considers that the adsorbed adenine can interact with the gold surface via the amine nitrogen (N10) and imidazole nitrogen (N7) according to Scheme 2a, or alternatively via the N10 and N1 nitrogen atoms as shown in, Scheme $2 \mathrm{~b}$. Both of these models have been previously postulated by other research groups. $40,41,42,43,44,45,46,47,48$

\section{- $\quad$ Scheme 2 -}

In the case of the interactions in Scheme 2a, the changes in the transition dipole angles with increasing electrode potential implies a counterclockwise rotation of the molecular plane around its normal direction. This rotation cannot be caused by the interaction between the permanent dipole of adenine, which has a magnitude 2.37 D and directed close to the C5-N7 bond direction, and the electric field at the interface, because the rotation decreases the magnitude of the dipole moment component in the direction of the permanent field. On the other hand, if the interactions between adenine and the metal involve the N10 and N1 atoms (shown in Scheme 2b) then changes in the $\varphi_{1640}$ and $\varphi_{1380}$ angles would involve a clockwise rotation in the 
molecular plane as the potential increases, the component of the molecular permanent dipole moment would increase in the direction of the electric field. Therefore, proposed orientation in Scheme $2 b$ is more consistent with the expected dipole-field interaction.

The proposed coordination of adenine to the metal through N10 and the N1 atoms also provide an explanation for the $\mathrm{pH}$ dependence that was previously observed. ${ }^{11}$ The adsorbing adenine molecule, from either acidic or neutral solutions, adopts the deprotonated structure, even at $\mathrm{pH}$ values below pKa regardless of the fact that the adenine in the bulk of solution consist solely of adenine molecules where the $\mathrm{N} 1$ atom is protonated. Therefore, the adsorption mechanism must involve the deprotonation of the N1 site in order to chemically interact with the metal surface.

A more quantitative picture of the evolution of the electrostatic interactions of adenine with the electric field can be obtained by evaluating the component of the molecular dipole moment in the electric field direction, $\mu_{z}$, as a function of the charge density of the electrode charge. The values of this component are related to the $\cos ^{2} \theta_{z}$ values, with $\theta_{z}$ being the angle between the permanent dipole of the molecule, $\mu_{o}$, and the static electric field direction. The values of $\cos ^{2} \theta_{z}$ have been obtained from $\alpha$ and either $\varphi_{1640}$ or $\varphi_{1380}$ and are plotted in Figure 7 as a function of the inverse of the surface charge density, $\sigma_{M}$, on the metal. The observed trend can be approximated by a linear decrease, which is expected for electrostatic interactions according to the Langevin model. ${ }^{49}$ For a freely rotating polar molecule in the presence of a static electric field, the Langevin-Debye equation predicts a linear decrease of $\cos ^{2} \theta_{z}$ with reciprocal of $\sigma_{M}$ : 


$$
\cos ^{2} \theta_{z}=\left(1-\frac{2 \varepsilon k_{B} T}{\mu_{o} \sigma_{M}}\right)
$$

where $\varepsilon$ is the dielectric constant and $k_{B} T$ is the thermal energy.

- $\quad$ Figure 7-

In Figure 7, a linear relationship is observed with a slope of ca. $-4.6 \mu \mathrm{C} \mathrm{cm}^{-2}$. Evidently, this value cannot be explained by eq. (7) if adopting the value of the electric permittivity of bulk water. This is not surprising as it is well known that organized water at the electrochemical interfaces adopts a much lower value (see, for instance reference $\left.{ }^{50}\right)$. Moreover, in the case of adsorbed adenine monolayer the potential drop takes place mostly across an ordered film of an organic dielectric. If the square of adenine refractive index, $n_{D 3 A d}$ in Figure 4 , is adopted for the dielectric constant in this case the value of the slope must be in the range -0.8 to $-1.5 \mu \mathrm{C} \mathrm{cm}^{-2}$, which is of the same order of magnitude than the experimental slope value.

\section{CONCLUSSIONS.}

A quantitative SNIFTIRS procedure has provided physical calculations of the angles between the normal direction to the electrode and the transition dipoles of two selected in-plane vibrations $\left(\theta_{1640}\right.$ and $\left.\theta_{1380}\right)$ of adenine adsorbed on $A u(111)$ electrodes. Using these values, the tilt angle, $\alpha$, of the molecular plane with respect to the electrode surface, and the two angles between each of the two selected transition dipoles and the projected normal direction to the electrode on the molecular plane, $\varphi_{1640}$ and $\varphi_{1380}$, have been evaluated at different potentials within the chemical 
adsorption potential range. The dependence of $\varphi_{1640}, \varphi_{1380}$ and $\alpha$ angles on the applied potential was determined.

The value of $\alpha$ is $\sim 40^{\circ}$ at potentials higher than the onset of adenine adsorption, in agreement with a $\mathrm{sp}^{3}$ hybridization of the $\mathrm{N} 10$ atom. This hybridization facilitates its coordination to the electrode, and suggests that the stabilization of the adsorbed monolayer is due to $\pi-\pi$ parallel displaced stacking interactions between adjacent adsorbed molecules.

The changes with potential of the $\varphi_{1640}$ and $\varphi_{1380}$ angles of the two selected transition dipoles were used to determine rotation of the molecular plane around its normal axis. These changes are consistent with coordination of the adenine molecule through the N10 and the N1 atoms with the metal surface. Thus, it is concluded that adenine adsorbs on the gold electrode with the N10 and N1 atoms directed towards the electrode surface and its molecular plane increasingly rotates over a range of about $16^{\circ}$ when increasing the static electric field intensity. This molecular organization provides an explanation about why the neutral deprotonated form of adenine is adsorbed at the gold surface even from solutions of very low $\mathrm{pH}$ values.

\section{ACKNOWLEDGEMENTS}

Financial support from the Spanish Ministry of Science and Technology (CTQ201019823), from the Spanish Ministry of Economy and Competitiveness (CTQ2014-57515C2-1-R), from the Junta de Andalucia (PAI FQM202) and a Discovery Grant from Natural Sciences and Engineering Council of Canada are gratefully acknowledged. 
The authors want to thank Prof. John Goddard and Dr Xueqin Ran for their valuable help in ab-initio calculations and Centro de Servicios de Informatica y Redes de Comunicaciones (CSIRC) of the University of Granada for the computing time.

\section{REFERENCES}

(1) Reiter, N. J.; Blad, H.; Abildgaard, F.; Butcher, S. E. Dynamics in the U6 RNA intramolecular stem-loop: A base flipping conformational changes. Biochemistry 2004, 43, 13739-13747.

(2) Andersen, A. A.; Collins, R. A. Rearrangement of a stable RNA secondary structure during VS ribozyme catalysis. Molecular Cell 2000, 5, 469-478.

(3) Huang, J. G.; Li, C.; Liang, Y. Q. FT-SERS studies on molecular recognition capabilities of monolayers of novel nucleolipid amphiphiles. Langmuir 2000, 16, 3937-3940.

(4) Berndt, P.; Kurihara, K.; Kunitake, T. MEASUREMENT OF FORCES BETWEEN SURFACES COMPOSED OF 2-DIMENSIONALLY ORGANIZED, COMPLEMENTARY AND NONCOMPLEMENTARY NUCLEOBASES. Langmuir 1995, 11, 3083-3091.

(5) Shimomura, M.; Nakamura, F.; ljiro, K.; Taketsuna, H.; Tanaka, M.; Nakamura, H.; Hasebe, K. Two-dimensional DNA-mimetic molecular organizations at the air-water interface. Journal of the American Chemical Society 1997, 119, 2341-2342.

(6) Kitano, H.; Ringsdorf, H. SURFACE BEHAVIORS OF NUCLEIC-ACID BASE-CONTAINING LIPIDS IN MONOLAYER AND BILAYER SYSTEMS. Bulletin of the Chemical Society of Japan 1985, 58, 2826-2828.

(7) Prado, C.; Prieto, F.; Rueda, M.; Feliu, J.; Aldaz, A. Adenine adsorption on Au(111) and $\mathrm{Au}(100)$ electrodes: Characterisation, surface reconstruction effects and thermodynamic study. Electrochimica Acta 2007, 52, 3168-3180.

(8) Alvarez-Malmagro, J.; Prieto, F.; Rueda, M.; Rodes, A. In situ Fourier transform infrared reflection absortion spectroscopy study of adenine adsorption on gold electrodes in basic media. Electrochimica Acta 2014, 140, 476-481.

(9) Chen, A. C.; Shi, Z. C.; Bizzotto, D.; Lipkowski, J.; Pettinger, B.; Bilger, C. lodide adsorption at the $\mathrm{Au}(111)$ electrode surface. Journal of Electroanalytical Chemistry 1999, 467, 342-353.

(10) Wu, S.; Lipkowski, J.; Magnussen, O. M.; Ocko, B. M.; Wandlowski, T. The driving force for (px root 3$)<->(1 \times 1)$ phase transition of $A u(111)$ in the presence of organic adsorption: a combined chronocoulometric and surface X-ray scattering study. Journal of Electroanalytical Chemistry 1998, 446, 67-77.

(11) Rueda, M.; Prieto, F.; Rodes, A.; Manuel Delgado, J. In situ infrared study of adenine adsorption on gold electrodes in acid media. Electrochimica Acta 2012, 82, 534-542.

(12) Rodes, A.; Rueda, M.; Prieto, F.; Prado, C.; Miguel Feliu, J.; Aldaz, A. Adenine Adsorption at Single Crystal and Thin-Film Gold Electrodes: An In Situ Infrared Spectroscopy Study. Journal of Physical Chemistry C 2009, 113, 18784-18794.

(13) Vaz-Dominguez, C.; Escudero-Escribano, M.; Cuesta, A.; Prieto-Dapena, F.; Cerrillos, C.; Rueda, M. Electrochemical STM study of the adsorption of adenine on $\mathrm{Au}(111)$ electrodes. Electrochemistry Communications 2013, 35, 61-64. 
(14) Broomhead, J. M. THE STRUCTURES OF PYRIMIDINES AND PURINES .2. A DETERMINATION OF THE STRUCTURE OF ADENINE HYDROCHLORIDE BY X-RAY METHODS. Acta Crystallographica 1948, 1, 324-329.

(15) Broomhead, J. M. THE STRUCTURES OF PYRIMIDINES AND PURINES .4. THE CRYSTAL STRUCTURE OF GUANINE HYDROCHLORIDE AND ITS RELATION TO THAT OF ADENINE HYDROCHLORIDE. Acta Crystallographica 1951, 4, 92-100.

(16) Rueda, M.; Prieto, F.; Alvarez-Malmagro, J.; Rodes, A. Evidences of adenine-thymine Interactions at gold electrodes interfaces as provided by in-situ infrared spectroscopy. Electrochemistry Communications 2013, 35, 53-56.

(17) Rueda, M.; Prieto, F.; Alvarez, J.; Rodes, A. Adenine-thymine interactions on gold electrodes studied by in-situ electrochemical FT-IR-spectroscopy. Febs Journal 2012, 279, 515516.

(18) Li, N. H.; Zamlynny, V.; Lipkowski, J.; Henglein, F.; Pettinger, B. In situ IR reflectance absorption spectroscopy studies of pyridine adsorption at the $\mathrm{Au}(110)$ electrode surface. Journal of Electroanalytical Chemistry 2002, 524, 43-53.

(19) Nichols, R. J.; Burgess, I.; Young, K. L.; Zamlynny, V.; Lipkowski, J. A quantitative evaluation of the adsorption of citrate on $\mathrm{Au}(111)$ using SNIFTIRS. Journal of Electroanalytical Chemistry 2004, 563, 33-39.

(20) Doneux, T.; Buess-Herman, C.; Hosseini, M. G.; Nichols, R. J.; Lipkowski, J. Adsorption of 2mercaptobenzimidazole on a Au(111) electrode. Electrochimica Acta 2005, 50, 4275-4282.

(21) Doneux, T.; Buess-Herman, C.; Lipkowski, J. Electrochemical and FTIR characterization of the self-assembled monolayer of 2-mercaptobenzimidazole on Au(111). Journal of

Electroanalytical Chemistry 2004, 564, 65-75.

(22) Su, Z.; Climent, V.; Leitch, J.; Zamlynny, V.; Feliu, J. M.; Lipkowski, J. Quantitative SNIFTIRS studies of (bi)sulfate adsorption at the Pt(111) electrode surface. Physical Chemistry Chemical Physics 2010, 12, 15231-15239.

(23) Alkire, R. C.; Kolb, D. M.; Lipkowski, J.; Ross, P. Advances in Electrochemical Science and Engineering. WILEY-VCH Verlag GmbH: Weinheim, 2006; $\mathrm{p} 315$.

(24) Allara, D. L.; Nuzzo, R. G. SPONTANEOUSLY ORGANIZED MOLECULAR ASSEMBLIES .1. FORMATION, DYNAMICS, AND PHYSICAL-PROPERTIES OF NORMAL-ALKANOIC ACIDS ADSORBED FROM SOLUTION ON AN OXIDIZED ALUMINUM SURFACE. Langmuir 1985, 1, 45-52. (25) Allara, D. L.; Swalen, J. D. AN INFRARED REFLECTION SPECTROSCOPY STUDY OF ORIENTED CADMIUM ARACHIDATE MONOLAYER FILMS ON EVAPORATED SILVER. Journal of Physical Chemistry 1982, 86, 2700-2704.

(26) Allara, D. L.; Baca, A.; Pryde, C. A. DISTORTIONS OF BAND SHAPES IN EXTERNAL REFLECTION INFRARED-SPECTRA OF THIN POLYMER-FILMS ON METAL SUBSTRATES. Macromolecules 1978, 11, 1215-1220.

(27) Clavilier, J.; Faure, R.; Guinet, G.; Durand, R. PREPARATION OF MONO-CRYSTALLINE PT MICROELECTRODES AND ELECTROCHEMICAL STUDY OF THE PLANE SURFACES CUT IN THE DIRECTION OF THE (111) AND (110) PLANES. Journal of Electroanalytical Chemistry 1980, 107, 205-209.

(28) Griffiths, P. R.; De Haseth, J. A. Fourier transform infrared spectrometry; Wiley: New York; Chichester, 1986. p xv, $656 \mathrm{p}$.

(29) Skoog, D. A.; Holler, F. J.; Crouch, S. R. Principles of instrumental analysis; 6th ed.; Thomson Brooks/Cole: Australia ; United Kingdom, 2007. p xv, 1039 p.

(30) Moskovits, M. SURFACE SELECTION-RULES. Journal of Chemical Physics 1982, 77, 44084416.

(31) Faguy, P. W.; Marinkovic, N. S. Design and performance of a new infrared reflection accessory for spectroelectrochemical studies. Applied Spectroscopy 1996, 50, 394-400. 
(32) Faguy, P. W.; Marinkovic, N. S.; Adzic, R. R. Infrared spectroscopic analysis of anions adsorbed from bisulfate-containing solutions on Pt(111) electrodes. Journal of

Electroanalytical Chemistry 1996, 407, 209-218.

(33) Faguy, P. W.; Marinkovic, N. S. SENSITIVITY AND REPRODUCIBILITY IN INFRARED SPECTROSCOPIC MEASUREMENTS AT SINGLE-CRYSTAL ELECTRODE SURFACES. Analytical Chemistry 1995, 67, 2791-2799.

(34) Faguy, P. W.; Fawcett, W. R. INFRARED REFLECTION-ABSORPTION SPECTROSCOPY OF THE ELECTRODE ELECTROLYTE SOLUTION INTERFACE - OPTICAL CONSIDERATIONS. Applied Spectroscopy 1990, 44, 1309-1316.

(35) Hansen, W. N. ELECTRIC FIELDS PRODUCED BY PROPAGATION OF PLANE COHERENT ELECTROMAGNETIC RADIATION IN A STRATIFIED MEDIUM. Journal of the Optical Society of America 1968, 58, 380-\&.

(36) Giese, B.; McNaughton, D. Surface-enhanced Raman spectroscopic and density functional theory study of adenine adsorption to silver surfaces. Journal of Physical Chemistry B 2002, 106, 101-112.

(37) Pettinger, B.; Lipkowski, J.; Hoon-Khosla, M. Simulation of SNIFTIRS experiments. Journal of Electroanalytical Chemistry 2001, 500, 471-478.

(38) Hunter, C. A.; Sanders, J. K. M. THE NATURE OF PI-PI INTERACTIONS. Journal of the American Chemical Society 1990, 112, 5525-5534.

(39) Sinnokrot, M. O.; Sherrill, C. D. Highly accurate coupled cluster potential energy curves for the benzene dimer: Sandwich, T-shaped, and parallel-displaced configurations. Journal of Physical Chemistry A 2004, 108, 10200-10207.

(40) Otto, C.; Demul, F. F. M.; Huizinga, A.; Greve, J. SURFACE ENHANCED RAMAN-SCATTERING OF DERIVATIVES OF ADENINE - THE IMPORTANCE OF THE EXTERNAL AMINO GROUP IN ADENINE FOR SURFACE BINDING. Journal of Physical Chemistry 1988, 92, 1239-1244.

(41) Chen, Q.; Frankel, D. J.; Richardson, N. V. Self-assembly of adenine on Cu(110) surfaces. Langmuir 2002, 18, 3219-3225.

(42) Taniguchi, I.; Umekita, K.; Yasukouchi, K. SURFACE-ENHANCED RAMAN-SCATTERING OF NICOTINAMIDE ADENINE-DINUCLEOTIDE (NAD+) ADSORBED ON SILVER AND GOLD ELECTRODES. Journal of Electroanalytical Chemistry 1986, 202, 315-322.

(43) Xiao, Y. J.; Chen, Y. F.; Gao, X. X. Comparative study of the surface enhanced near infrared Raman spectra of adenine and NAD(+) on a gold electrode. Spectrochimica Acta Part aMolecular and Biomolecular Spectroscopy 1999, 55, 1209-1218.

(44) Xiao, Y. J.; Markwell, J. P. Potential dependence of the conformations of nicotinamide adenine dinucleotide on gold electrode determined by FT-near-IR-SERS. Langmuir 1997, 13, 7068-7074.

(45) Xiao, Y. J.; Wang, T.; Wang, X. Q.; Gao, X. X. Surface-enhanced near-infrared Raman spectroscopy of nicotinamide adenine dinucleotides on a gold electrode. Journal of Electroanalytical Chemistry 1997, 433, 49-56.

(46) Demers, L. M.; Ostblom, M.; Zhang, H.; Jang, N. H.; Liedberg, B.; Mirkin, C. A. Thermal desorption behavior and binding properties of DNA bases and nucleosides on gold. Journal of the American Chemical Society 2002, 124, 11248-11249.

(47) McNutt, A.; Haq, S.; Raval, R. RAIRS investigations on the orientation and intermolecular interactions of adenine on $\mathrm{Cu}(110)$. Surface Science 2003, 531, 131-144.

(48) Damian, A.; Omanovic, S. Interactive adsorption behavior of $N A D(+)$ at a gold electrode surface. Langmuir 2007, 23, 3162-3171.

(49) Langevin, P. Magnetism and theory of electrons. Annales De Chimie Et De Physique 1905, $5,70-127$. 
(50) Bange, K.; Straehler, B.; Sass, J. K.; Parsons, R. THE INTERACTION OF BR WITH AG(110) COMPARISON OF ELECTROCHEMICAL AND GAS-PHASE ADSORPTION MEASUREMENTS. Journal of Electroanalytical Chemistry 1987, 229, 87-98. 


\section{LEGENDS}

Scheme 1.- Representation of an adsorbed adenine molecule on the $\mathrm{Au}(111)$ electrode with a tilt angle of the molecular plane, $\alpha$, relative to the normal direction of the electrode surface. $\varphi_{1640}$ and $\varphi_{1380}$ are the angles between the corresponding transition dipoles vibrations and the projected normal to the electrode on the molecular plane.

Scheme 2.- Representation of the adenine molecular plane adsorbed on $\mathrm{Au}(111)$ electrodes. The projected normal direction to the electrode on the molecular plane (black dashed line), the approximate directions of the transition dipoles of the vibrations at 1380 (green dashed line) and $1640 \mathrm{~cm}^{-1}$ (red dashed line), respectively. The blue arrow represents the permanent molecular dipole direction. The interaction of the adenine molecule with the metal surface via the a) N10 and N7 atoms or b) the $\mathrm{N} 10$ and N1 atoms.

Figure 1.-Transmission absorption spectrum of $10 \mathrm{mM}$ adenine in a) $0.5 \mathrm{M} \mathrm{NaF} / \mathrm{D}_{2} \mathrm{O}$ and b) $0.5 \mathrm{M} \mathrm{NaF} / \mathrm{H}_{2} \mathrm{O}$.

Figure 2.- The p-polarized SNIFTIR spectra of deuterated adenine adsorbed on Au(111) electrodes in $2 \mathrm{mM}$ solutions in $0.5 \mathrm{M} \mathrm{NaF} / \mathrm{D}_{2} \mathrm{O}$ at selected sample potentials with a reference potential of $-0.65 \mathrm{~V}$ vs. $\mathrm{Ag} / \mathrm{AgCl}$. The s-polarized SNFITIR spectrum at $0.05 \mathrm{~V}$ vs $\mathrm{Ag} / \mathrm{AgCl}$ is included for the comparison.

Figure 3.- a) The experimental p-polarized (blue dashed line)and s-polarized (red solid line) SNIFTIR spectra around the bands at 1380 and $1640 \mathrm{~cm}^{-1}$ of deuterated adenine adsorbed on $\mathrm{Au}(111)$ electrodes from $2 \mathrm{mM}$ solutions in $0.5 \mathrm{M} \mathrm{NaF} / \mathrm{D}_{2} \mathrm{O}$ at $-0.11 \mathrm{~V}$ vs. 
$\mathrm{Ag} / \mathrm{AgCl}$ and the corrected p-polarized SNIFTIR spectrum according to Equation (3) (red solid line). b) The simulated SNIFTIRS bands at 1380 and $1640 \mathrm{~cm}^{-1}$ for an adenine film consisting of randomly oriented molecules with a surface concentration of $2.2 \times 10^{-10}$ $\mathrm{mol} \mathrm{cm}{ }^{-2}$.

Figure 4.- a) Refractive index and b) attenuation coefficient of deuterated adenine for the 1640 and $1380 \mathrm{~cm}^{-1}$ wavenumber regions.

Figure 5.- Potential dependence of the transition dipole angles of the $1640 \mathrm{~cm}^{-1}(\bullet)$ and $1380 \mathrm{~cm}^{-1}(\Delta)$ vibrations relative to the normal direction of the electrode surface for an adsorbed deuterated adenine film. The schematic representations of the transition dipole directions in the molecular plane are included.

Figure 6- .- a) Angle of the molecular plane of adsorbed adenine as a function of the potential. b) The potential dependence of the angles relative to the projected normal direction of the electrode within the molecular plane of the transition dipoles moments of the $1640 \mathrm{~cm}^{-1}(\bullet)$ and $1380 \mathrm{~cm}^{-1}(\Delta)$ vibrations of deuterated adenine adsorbed on $\mathrm{Au}(111)$. The error bars account for 5 degrees of uncertainty in the calculation of the angle between the transition dipoles of the vibrations at 1640 and $1380 \mathrm{~cm}^{-1}$.

Figure 7.-The cosine square of the angle between the permanent dipole moment of an adsorbed adenine film and the static electric field with respect to the inverse of the charge density on the metal surface . 